\title{
Constellation Labeling Maps for Low Error Floors
}

\author{
Don Torrieri and Matthew C. Valenti
}

\begin{abstract}
A constellation labeling map is the assignment of a bit pattern to each symbol in a signal-set constellation. In a system with iterative decoding and demodulation, the error floor of the bit error rate is highly dependent on the labeling map. A simple class of labeling maps that significantly lower the error floors is presented. Examples show the applications of the proposed mapping to multiple phase-shift keying (MPSK), quadrature amplitude modulation (QAM), and continuous-phase frequency-shift keying (CPFSK). Simulation results indicate that the proposed labeling maps are comparable to or better than other labeling maps in providing a low error floor. A major advantage of the proposed labeling maps is that they are easily generated even when the alphabet size is large.
\end{abstract}

Index Terms - Constellation, labeling map, error floor, iterative decoding, bit-interleaved coded modulation.

\section{INTRODUCTION}

$\mathbf{M}$ ANY communication systems include iterative decoding and demodulation in which soft-decision information is exchanged between the demodulator and the decoder, which itself may be internally iterative. For example, a system with bit-interleaved coded-modulation (BICM), which is used to mitigate fading [1], may also use iterative decoding and demodulation (BICM-ID system) [2], [3], [4]. The iterative decoding and demodulation minimizes any performance degradation experienced by BICM over the additive white Gaussian noise (AWGN) channel.

Plots of the bit error rate for systems with iterative decoding and demodulation generally exhibit a waterfall region, which is characterized by a rapid decrease in the bit error rate as the signal-to-noise ratio increases, and an error-floor region, in which the bit error rate decreases much more slowly. The choice of the labeling map has a major impact on both regions. In this paper, we present a simple method for constructing labeling maps that produce low error floors for an arbitrary constellation and an error-control code that uses iterative decoding and demodulation. A low error floor may be important for radio-relay communications, space-ground communications, or when an automatic-repeat request is not feasible because of the variable delays.

Methods for generating good labeling maps for low error floors have been previously described [5], [6], [7]. These methods entail computer searches based on approximate upper bounds on the bit error rate. The new labeling maps presented

Manuscript received November 19, 2007; revised March 8, 2008 and June 30, 2008; accepted August 28, 2008. The associate editor coordinating the review of this paper and approving it for publication was J. Tugnait.

D. Torrieri is with the US Army Research Laboratory, 2800 Powder Mill Road, Adelphi, MD 20783 (e-mail: dtorr@arl.army.mil).

M. C. Valenti is with West Virginia University.

This paper was presented in part at the IEEE Military Communications Conference (MILCOM), Orlando, FL, Oct. 2007.

Digital Object Identifier 10.1109/T-WC.2008.071276 in this paper are based on the Euclidean distances in the signal-set constellations and are much simpler to generate. Simulation results indicate that the proposed labeling maps are comparable to or better than other labeling maps in providing a low error floor.

In addition to a suitable choice of the labeling map, there are several independent strategies for lowering the error floor. One can lower the error floor by strengthening or appropriately selecting the error-control code. As examples, one can use a turbo code instead of a convolutional code or more powerful component codes within the turbo code. One can use a regular low-density parity-check (LDPC) code instead of a comparable irregular one to lower the error floor. Bit interleavers that ensure the unequal protection of bits can be designed to provide low error floors for both bit-interleaved turbo-coded systems [8] and LDPC-coded systems [9]. The new labeling maps can supplement any of the other methods of lowering the error floor and lower it further.

A Gray labeling map minimizes the number of bit errors that occur if an adjacent symbol of a received symbol is assigned the highest likelihood or largest metric by the decoder. Thus, a Gray labeling map will provide an early onset of the waterfall region, but produces a relatively high error floor primarily determined by the minimum Euclidean distance of the symbol set. In contrast, the new labeling map described in Section II lowers the error floor at the cost of an adversely shifted waterfall region.

\section{LABELING MAPS}

The major components of a communication system with iterative decoding and demodulation are diagrammed in Fig. 1. The interleaver and deinterleaver are omitted if the system does not use BICM. In the transmitter, message bits are encoded, bit-interleaved, and then applied to the modulator. A constellation labeling or labeling map is the mapping of a bit pattern to each symbol or point in a signal-set constellation. Each set of $m=\log _{2} q$ consecutive bits in the input $\mathbf{b}=\left\{b_{0}, \ldots, b_{m-1}\right\} \in[0,1]^{m}$ is mapped into a $q$-ary symbol $\mathbf{s}=\mu(\mathbf{b})$, where $\mu(\mathbf{b})$ is the labeling map, and the set of constellation symbols has cardinality $q$. In the receiver, the demodulator converts the received signal into a sequence of received symbols. A demapper within the demodulator processes each received symbol to produce a vector of bit metrics. This vector provides extrinsic information that is deinterleaved and passed to the decoder. The demapper and decoder exchange extrinsic information until bit decisions are made by the decoder after a specified number of iterations.

The Euclidean distance is a measure of the separation between two constellation points. An adjacent constellation symbol of symbol $i$ is one at the minimum Euclidean distance 


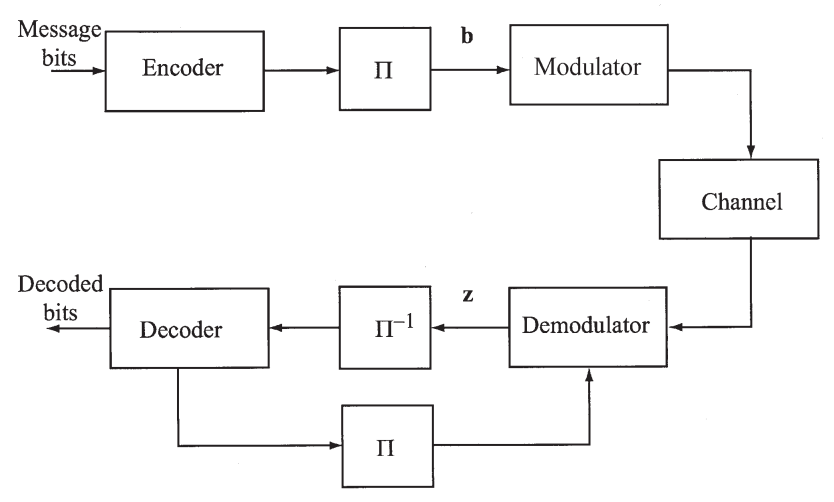

Fig. 1. Communication system with iterative decoding and demodulation. $\Pi$ denotes an interleaver, and $\Pi^{-1}$ denotes a deinterleaver. The interleavers and deinterleaver are omitted if the system does not use BICM.

$d_{e 1}(i)$ from symbol $i$. A second closest constellation symbol of symbol $i$ is one at the second shortest Euclidean distance $d_{e 2}(i)$ from symbol $i$. A set of bits is said to be essentially known if the decoder has assigned them very high likelihood ratios that are fed back to the demodulator.

Let $\mathbf{s}_{i}, 1 \leq i \leq q$, denote symbol $i$ of the constellation, and $b_{k}\left(\mathbf{s}_{i}\right)$ denote bit $k$ of $\mathbf{s}_{i}$. Consider the constellation symbols and the corresponding bits produced by the decoder. When the SNR is high enough and there are enough decoder iterations, some bits are essentially known by the decoder. The constellation symbols that include bits equal to the essentially known bits constitute a subset $A$ of the constellation. For example, if the first bit of an 8 -symbol constellation is essentially known to be $b$, then the 4 constellation symbols with $b_{1}\left(\mathbf{s}_{i}\right)=b$ are in $A$. Consider a demodulator iteration after the demodulator receives extrinsic information from the decoder. Let $\mathbf{y}=\alpha \mathbf{s}+\mathbf{n}$ denote an arbitrary received symbol, where $\mathbf{s}$ is the transmitted symbol, $\alpha$ is the fading amplitude, and $\mathbf{n}$ is complex Gaussian noise with variance $N_{0} / 2$ per component. If a bit is essentially known, the bit metric produced by the demodulator for that bit no longer significantly affects the computation of the decoder metric. However, if a bit is unknown, then the demodulator bit metric has a significant effect on the decoder bit metric produced by the next decoder iteration. Let $v_{j}=\log \left\{\operatorname{Pr}\left[b_{j}=1\right] / \operatorname{Pr}\left[b_{j}=0\right]\right\}$ denote the extrinsic log-likelihood ratio for bit $j$ that is produced by the decoder and fed back to the demodulator, where $\operatorname{Pr}[\cdot]$ denotes a probability. The demodulator metric for bit $k$ is [10] , [11]

$$
z_{k}=\log \frac{\sum_{\mathbf{s}_{i} \in \mathcal{D}_{k}^{(1)}} p\left(\mathbf{y} \mid \mathbf{s}_{i}\right) \prod_{\substack{j=0 \\ j \neq k}}^{m-1} \exp \left[b_{j}\left(\mathbf{s}_{i}\right) v_{j}\right]}{\sum_{\mathbf{s}_{i} \in \mathcal{D}_{k}^{(0)}} p\left(\mathbf{y} \mid \mathbf{s}_{i}\right) \prod_{\substack{j=0 \\ j \neq k}}^{m-1} \exp \left[b_{j}\left(\mathbf{s}_{i}\right) v_{j}\right]}
$$

where $p\left(\mathbf{y} \mid \mathbf{s}_{i}\right)$ is the conditional density of $\mathbf{y}$ given that $\mathbf{s}_{i}$ was transmitted and $\mathcal{D}_{k}^{(b)}=\left\{\mathbf{s}_{i}: b_{k}\left(\mathbf{s}_{i}\right)=b\right\}$ contains all symbols labeled with $b_{k}=b$. As an example, suppose that all bits are essentially known. Then (1) indicates that $z_{k}$, which will not significantly affect subsequent decoder computations, is approximately equal to $\log \left[p\left(\mathbf{y} \mid \mathbf{s}_{n}\right) / p\left(\mathbf{y} \mid \mathbf{s}_{m}\right)\right]$, where $\mathbf{s}_{n}$ and $\mathbf{s}_{m}$ both include $m-1$ of the essentially known bits but $b_{k}\left(\mathbf{s}_{n}\right) \neq b_{k}\left(\mathbf{s}_{m}\right)$.

Consider two terms in one of the summations in (1). In term $t_{l}, \mathbf{s}_{l} \in \mathcal{D}_{k}^{(b)} \cap A$, and in term $t_{m}, \mathbf{s}_{m} \in \mathcal{D}_{k}^{(b)} \cap A^{c}$, where $A^{c}$ denotes the complement of $A$. Suppose that for $n \neq k$, bit $n$ is essentially known so that $v_{n}$ has a large magnitude. If $b_{n}\left(\mathbf{s}_{l}\right) \neq b_{n}\left(\mathbf{s}_{m}\right)$, then the factor $\exp \left[b_{n}\left(\mathbf{s}_{l}\right) v_{n}\right]$ in $t_{l}$ is much larger than the factor $\exp \left[b_{n}\left(\mathbf{s}_{m}\right) v_{n}\right]$ in $t_{m}$. Therefore, in both summations in (1), any term for which $\mathbf{s}_{i} \notin \mathcal{D}_{k}^{(b)} \cap A$ is negligible compared with terms for which $\mathbf{s}_{i} \in \mathcal{D}_{k}^{(b)} \cap A$. For an unknown bit $k$,

$$
z_{k} \simeq \log \frac{\sum_{\mathbf{s}_{i} \in \mathcal{D}_{k}^{(1)} \cap A} p\left(\mathbf{y} \mid \mathbf{s}_{i}\right) \prod_{\substack{j=0 \\ j \neq k}}^{m-1} \exp \left[b_{j}\left(\mathbf{s}_{i}\right) v_{j}\right]}{\sum_{\mathbf{s}_{i} \in \mathcal{D}_{k}^{(0)} \cap A} p\left(\mathbf{y} \mid \mathbf{s}_{i}\right) \prod_{\substack{j=0 \\ j \neq k}}^{m-1} \exp \left[b_{j}\left(\mathbf{s}_{i}\right) v_{j}\right]} .
$$

If a labeling map can ensure that the set $A$ does not include symbols that are adjacent in the constellation, then (2) indicates that each $z_{k}$ for an unknown bit $k$ is independent of adjacent symbols at the minimum distance $d_{e 1}(i)$ and, hence, the effective minimum distance for the demodulator iteration is at least $d_{e 2}(i)$. Thus, if the minimum distance predominates in determining bit errors, then subsequent decoder iterations with this labeling map are more likely to correctly determine the bits and lower the error floor than iterations with maps for which set $A$ includes adjacent symbols.

A TV labeling map is defined as a labeling map for a constellation with $m \geq 3$ such that adjacent symbols are absent from any set of symbols with $\kappa$ known bits if $2 \leq \kappa \leq m-1$.

Proposition 1: A labeling map for a constellation with $m \geq$ 3 is a TV labeling map if and only if the minimum Hamming distance $d_{a}(i)$ from a constellation symbol $i$ to its adjacent ones is at least $m-1$.

Proof: Necessity. Assume that a labeling map is such that some symbol $i$ is at Hamming distance $d_{a}(i) \leq m-2$ from one or more adjacent ones. Then at least two bits are common to the symbol $i$ and one or more of its adjacent symbols. If the common bits are known, symbol $i$ and one or more of its adjacent symbols are members of the set of symbols with the two known bits. Thus, the labeling map cannot be a TV labeling map. Sufficiency. Assume that a labeling map has $d_{a}(i) \geq m-1$ for any symbol $i$. Let $d_{2}(i, A)$ denote the Hamming distance between symbol $i$ and another member of a set $A$ of symbols with $\kappa$ known bits. If $2 \leq \kappa \leq m-1$, then $d_{2}(i, A) \leq m-\kappa<m-1 \leq d_{a}(i)$ for any symbol $i$. Since $d_{2}(i, A)<d_{a}(i)$, set $A$ cannot include both symbol $i$ and its adjacent symbols.

The proposition implies that a TV labeling map can be constructed by assigning bit patterns to symbols such that the Hamming distance to adjacent symbols always is at least $m-1$. In the following labeling algorithm, the symbols are labeled sequentially. After each symbol labeling, the unused bit patterns are called the remaining labels.

Labeling Algorithm: Two tables are associated with each symbol $i$. The adjacent-symbol table $S(i)$ is a list of symbols that are adjacent to symbol $i$. The adjacent-label table $L(i)$ is a list of labels that could be used by adjacent symbols. This table is initially empty. After symbol $i$ is labeled, $L(i)$ is a list of remaining labels that are at Hamming distance $m-1$ 
or $m$ from the label of symbol $i$. As successive symbols are labeled, $L(i)$ is shortened. One symbol is selected to be the first labeled symbol and is labeled arbitrarily.

Using the adjacent-symbol tables, arbitrarily select one of the unlabeled symbols that have the largest number of labeled adjacent symbols. This selected symbol is labeled with one of the remaining labels that is common to all the adjacentlabel tables of its labeled adjacent symbols. If there is more than one such label, one of them is chosen arbitrarily. The process terminates when every symbol has been labeled. If at any step, no further symbol labeling is possible, then the algorithm returns to the last arbitrary choice, erases all labels subsequent to this choice, makes a different choice, and then continues with the sequential labeling.

A TV labeling map exists only if every constellation symbol has $m+1$ or fewer adjacent symbols. Many TV labeling maps exist for most practical constellations. If an $m$-digit binary number is modulo- 2 added to all the bit labels of one TV labeling map, then another TV labeling map is produced. Furthermore, rotated and reflected versions of a TV labeling map can be constructed.

\section{EXAMPLES OF LABELING MAPS}

Example 1: For multiple phase-shift keying (MPSK), let $\mathbf{s}_{i}=\exp (j 2 \pi i / q), i=0,1, \ldots, q-1$, denote the complex value of constellation symbol $i$, where $j=\sqrt{-1}$ and $q=2^{m}$. For the small alphabet with $m=3$, some previously described labeling maps [3], [7] belong to the class of TV labeling maps. For $m=3$, a TV labeling map is the following.

\begin{tabular}{|c|c|c|c|}
\hline symbol & bit label & symbol & bit label \\
\hline 0 & 010 & 4 & 110 \\
\hline 1 & 001 & 5 & 101 \\
\hline 2 & 100 & 6 & 000 \\
\hline 3 & 011 & 7 & 111 \\
\hline
\end{tabular}

The minimum Hamming distance between symbols 0 and 7 and all other adjacent symbols is equal to or greater than 2 . Suppose that the first two bits of the map are essentially known to be 10 . Then the decoder will use the demodulator metrics for symbol 2 and symbol 5, which are not adjacent symbols, to make a decision on the third bit. For $m=4$, a TV labeling map is the following.

\begin{tabular}{|c|c|c|c|}
\hline symbol & bit label & symbol & bit label \\
\hline 0 & $\begin{array}{lllllllllllll} & 0 & 0\end{array}$ & 8 & $\begin{array}{llllll}0 & 1 & 1 & 0\end{array}$ \\
\hline 1 & $\begin{array}{llllll}0 & 1 & 1 & 1\end{array}$ & 9 & $\begin{array}{lllllll}0 & 0 & 0 & 1\end{array}$ \\
\hline$\overline{2}$ & $\begin{array}{lllll} & 0 & 0 & 1\end{array}$ & 10 & $\begin{array}{llll} & 1 & 1 & 1\end{array}$ \\
\hline 3 & 11110 & 11 & $\begin{array}{llll}1 & 0 & 0 & 0\end{array}$ \\
\hline$\overline{4}$ & $\begin{array}{lllll}0 & 0 & 1 & 1\end{array}$ & 12 & $\begin{array}{lllll}0 & 1 & 0 & 1\end{array}$ \\
\hline$\overline{5}$ & $\begin{array}{lllllll}0 & 1 & 0 & 0\end{array}$ & 13 & $\begin{array}{llllll}0 & 0 & 1 & 0\end{array}$ \\
\hline 6 & $\begin{array}{llll}1 & 0 & 1 & 0\end{array}$ & 14 & 1100 \\
\hline 7 & 11101 & 15 & $\begin{array}{llll}1 & 0 & 1 & 1\end{array}$ \\
\hline
\end{tabular}

Example 2: In Fig. 2 for quadrature amplitude modulation (QAM), the bit labels of a TV labeling map for the 16QAM constellation are expressed in a decimal format. The complex-valued symbols have 2, 3, or 4 adjacent symbols. The application of the labeling algorithm is illustrated by its first few steps. The constellation symbols are denoted by $0,1, \ldots, 15$ from left to right and from top to bottom starting in the upper

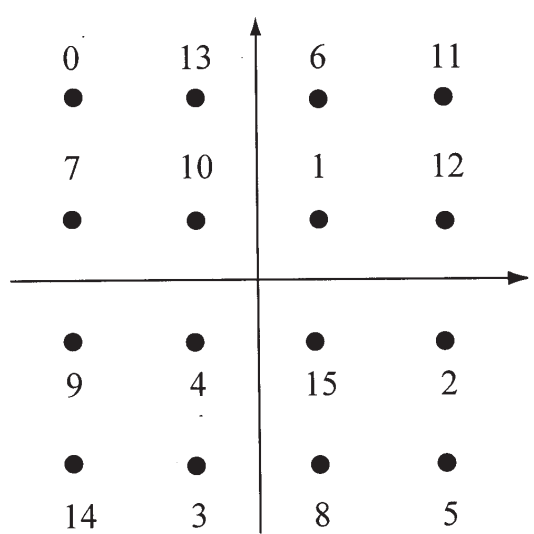

Fig. 2. Labeling map for 16-QAM constellation.

\begin{tabular}{cccc|cccc}
3 & 52 & 27 & 44 & 17 & 38 & 9 & 62 \\
$\bullet$ & $\bullet$ & $\bullet$ & $\bullet$ & $\bullet$ & $\bullet$ & $\bullet$ & $\bullet$ \\
28 & 43 & 4 & 51 & 14 & 57 & 22 & 33 \\
$\bullet$ & $\bullet$ & $\bullet$ & $\bullet$ & $\bullet$ & $\bullet$ & $\bullet$ & $\bullet$ \\
39 & 16 & 63 & 8 & 53 & 2 & 45 & 26 \\
$\bullet$ & $\bullet$ & $\bullet$ & $\bullet$ & $\bullet$ & $\bullet$ & $\bullet$ & $\bullet$ \\
56 & 15 & 32 & 23 & 42 & 29 & 50 & 5 \\
$\bullet$ & $\bullet$ & $\bullet$ & $\bullet$ & $\bullet$ & $\bullet$ & $\bullet$ & $\bullet$ \\
\hline$\bullet$ & $\bullet$ & $\bullet$ & $\bullet$ & $\bullet$ & $\bullet$ & $\bullet$ & $\bullet$ \\
6 & 49 & 30 & 41 & 20 & 35 & 12 & 59 \\
$\bullet$ & $\bullet$ & $\bullet$ & $\bullet$ & $\bullet$ & $\bullet$ & $\bullet$ & $\bullet$ \\
25 & 46 & 1 & 54 & 11 & 60 & 19 & 36 \\
$\bullet$ & $\bullet$ & $\bullet$ & $\bullet$ & $\bullet$ & $\bullet$ & $\bullet$ & $\bullet$ \\
34 & 21 & 58 & 13 & 48 & 7 & 40 & 31 \\
$\bullet$ & $\bullet$ & $\bullet$ & $\bullet$ & $\bullet$ & $\bullet$ & $\bullet$ & $\bullet$ \\
61 & 10 & 37 & 18 & 47 & 24 & 55 & 0
\end{tabular}

Fig. 3. Labeling map for 64-QAM constellation.

left corner of the figure. Symbol 0 is labeled 0000 . Table $L(0)$ lists the labels 1111, 1110, 1101, 1011, and 0111. Since both $S(1)$ and $S(4)$ list symbol 0 , symbol 1 is arbitrarily selected to be the next labeled symbol, and 1101 (13) is arbitrarily chosen from $L(0)$ as its label. This label is deleted from $L(0)$. Symbol 4 is arbitrarily selected to be the next labeled symbol, and 0111 (7) is arbitrarily chosen from $L(0)$ as its label. Symbol 5 is the only unlabeled symbol with 2 labeled adjacent symbols, so it is the next labeled symbol. Since 1010 is the only label common to both $L(1)$ and $L(4)$, symbol 5 is labeled 1010 (10). The labeling algorithm is continued until we obtain the TV labeling map of Fig. 2.

The "D5 mapping" of [12], which is proposed as an approximation of an anti-Gray map, belongs to the class of TV labeling maps for 64-QAM. In Fig. 3, the bit labels of a TV labeling map for the 64-QAM constellation are expressed in a decimal format. 
Example 3: Consider a noncoherent $q$-ary continuousphase frequency-shift keying (CPFSK) system with modulation index $h>0$ that does not exploit the memory due to the continuous phase of the signal. Then the signal-set constellation consists of $q$ symbols representing the signals $s_{l}(t)=e^{j 2 \pi l h t / T} / \sqrt{T}, 0 \leq t \leq T, l=1,2, \ldots, q$, where $T$ is the signal duration. The square of the Euclidean distance between symbols $i$ and $j$ is

$$
\begin{aligned}
d^{2}(|i-j|) & =\int_{0}^{T}\left|s_{i}(t)-s_{j}(t)\right|^{2} d t \\
& =2 \mathcal{E}_{s}\left[1-\frac{\sin [(i-j) h \pi]}{(i-j) h \pi}\right]
\end{aligned}
$$

where $\mathcal{E}_{s}$ is the signal energy, and the Euclidean distance is a function of $|i-j|$ because $\sin x / x$ is an even function of $x$.

If $0<h<1$, an adjacent symbol corresponds to the signal closest in frequency to the signal associated with a specified symbol, which is true because the minimum Euclidean distance between constellation symbols is $d(1)<d(k)$ for any integer $k \geq 2$. The latter follows from the fact that $\sin h \pi / h \pi>\sin k h \pi / k h \pi, 0<h<1$, for $k \geq 2$, which may be proved by showing that $k \sin h \pi-\sin k h \pi>0$, $0<h<1$, for $k \geq 2$. The proof is by mathematical induction. Assume that $0<h<1$. Since $\sin 2 h \pi=$ $2 \sin h \pi \cos h \pi$, the inequality is true for $k=2$. Assume that $k \sin h \pi-\sin k h \pi>0$ is true for some $k \geq 2$. Using this inequality, we obtain $(k+1) \sin h \pi-\sin (k+1) h \pi=$ $(k+1) \sin h \pi-\sin k h \pi \cos h \pi-\sin h \pi \cos k h \pi>\sin h \pi(k+$ $1-k \cos h \pi-\cos k h \pi)>0$, which completes the proof. Since $d(1)$ is the minimum Euclidean distance between constellation symbols, each symbol has either one or two adjacent symbols.

The TV labeling maps for $m=3$ and $m=4$ in example 1 are applicable to noncoherent CPFSK with $q=8$ and $q=16$, respectively. For $q=8$, symbol $i$ has adjacent symbols $i-1$ and $i+1,1 \leq i \leq 6$, symbol 0 has adjacent symbol 1 , and symbol 7 has adjacent symbol 6. Symbols 0 and 7 are not adjacent. Since symbol 6 is the only symbol adjacent to symbol 7, the labeling map indicates that $d_{a}(7)=3$.

\section{Characterisics of TV Labeling Maps}

If all the bits in a symbol are known except one, then the dominant influence on the bit error probability over the AWGN channel is the minimum Euclidean distance between the two constellation symbols that differ in that one bit [1], [3]. Thus, the asymptotic error floor of the bit error probability is determined by the minimum Euclidean distance $D_{e}$ between constellation symbols that differ in a single bit of their labels. The asymptotic error floor with its extremely low bit error probability is unlikely to be reached by practical communication systems. The error floor that is minimized by a TV labeling map is reached when at least two decoded bits are essentially known. However, within the class of TV labeling maps for a constellation, maps with the largest value of $D_{e}$ are found to provide the lowest error floors for both the AWGN and Rayleigh channels. Labeling maps that provide low error floors for the AWGN channel are also well suited for the Rayleigh channel [5].

A regular constellation is one in which all adjacent symbols are separated by the same Euclidean distance $d_{e 1}$ and there is a path between any two symbols that passes through successive adjacent symbols. The MPSK, QAM, and noncoherentCPFSK constellations are regular constellations. Consider a regular constellation with a TV labeling map and symbols $s_{1}$ and $s_{2}$ with bit patterns that differ in a single bit and do not differ in $m-1$ identical bits. Let $\beta$ denote the number of branches in a path from $s_{1}$ to $s_{2}$ that passes through successive adjacent symbols. For example, starting from the symbol labeled 0 in Fig. 2, the symbol labeled 1, which differs in a single bit of its label, can be reached by a path passing through the 3 successive adjacent symbols labeled 13,6, and 1 , and $\beta=3$ for this path.

Proposition 2: If a TV labeling map of a regular constellation has some adjacent symbols that differ in $m$ bits, then $\beta=2$ for some paths between symbols that differ in a single bit. If $m$ is an odd number, a TV labeling map of a regular constellation must have some adjacent symbols that differ in $m$ bits, and $\beta=2$ for some paths between symbols that differ in a single bit. A TV labeling map of a QAM constellation with an odd $m$ or some adjacent symbols that differ in $m$ bits has $D_{e}=\sqrt{2} d_{e 1}$.

Proof: Suppose that symbol $s_{2}$ has adjacent symbols $s_{1}$ and $s_{3}$. If $s_{2}$ has $m$ bits that differ from $s_{1}$ and $m-1$ bits that differ from $s_{3}$, then $s_{3}$ has only a single bit that differs from $s_{1}$. Therefore, $\beta=2$ for the path through $s_{1}, s_{2}$, and $s_{3}$, and the first statement of the proposition is proved. Each of the identical bits must change an even number of times as a path between symbols $s_{1}$ and $s_{2}$ that differ in a single bit is traversed through successive adjacent symbols. Thus, the total number of bit changes must be an odd number. If $m$ is an odd number and no adjacent symbols differ in $m$ bits, then the total number of bit changes also must equal $(m-1) \beta$, which is an even number. This contradiction proves the second statement of the proposition. For a QAM constellation, the Pythagorean theorem implies that $D_{e}=\sqrt{2} d_{e 1}$.

Proposition 3: If $m$ is an even number and a TV labeling map of a regular constellation has adjacent symbols that differ in exactly $m-1$ bits, then $\beta \geq m-1$. A TV labeling map of a square QAM constellation with an even $m$ and adjacent symbols that differ in exactly $m-1$ bits has

$$
D_{e} \geq\left(\frac{m^{2}}{2}-m+1\right)^{1 / 2} d_{e 1} .
$$

Proof: Each of the identical bits must change an even number of times as a path between symbols $s_{1}$ and $s_{2}$ that differ in a single bit is traversed through successive adjacent symbols. If $\beta$ is an odd number, each of the $m-1$ identical bits must be unchanged in traversing at least one branch of the path. Since adjacent symbols separated by a branch have at most one bit that is unchanged, $\beta \geq m-1$. Suppose that $m$ is an even number and the TV labeling map has adjacent symbols that differ in exactly $m-1$ bits. The total number of bit changes in a path from $s_{1}$ to $s_{2}$ must be an odd number and also must equal $(m-1) \beta$. Therefore, $\beta$ must be an odd number, and hence $\beta \geq m-1$. For a square QAM constellation with an even $m$, the minimum Euclidean distance for a path with $\beta=m-1$ occurs when there are $m / 2$ branches in one direction and $m / 2-1$ branches in the orthogonal direction. Since $\beta \geq m-1$, the Pythagorean theorem implies (4). 
Some TV labeling maps may have relatively low values of $\beta$ and $D_{e}$. However, if $m$ is an even number, the constrained labeling algorithm prevents the occurrence of this undesirable feature by constraining each adjacent-channel table $L(i)$ to have labels only at Hamming distance $m-1$ from the symbol $i$.

Simulation experiments indicate that within the class of TV labeling maps for a constellation, those with the largest value of $D_{e}$ provide the lowest error floors. For the Rayleigh channel, further distinctions within the class of TV labeling maps with the largest value of $D_{e}$ can be made by calculating the harmonic mean of the squared Euclidian distances of signals with labels that differ in just one bit position [1], [2], [3]. The harmonic mean is an approximate measure of the horizontal offset of a bit-error-rate curve. For the Rayleigh channel and a $q$-ary constellation with a labeling map, the harmonic mean is

$$
\xi=\left(\frac{1}{m 2^{m}} \sum_{k=1}^{m} \sum_{b=0}^{1} \sum_{\mathbf{s}_{i} \in \mathcal{D}_{k}^{(b)}} \frac{1}{\left\|\mathbf{s}_{i}-\widetilde{\mathbf{z}}_{k}\left(\mathbf{s}_{i}\right)\right\|^{2}}\right)^{-1}
$$

where $m=\log _{2} q$ and $\widetilde{\mathbf{z}}_{k}\left(\mathbf{s}_{i}\right)$ is the symbol whose label has the same bit pattern as $\mathbf{s}_{i}$ except at bit $k$. Although the harmonic mean depends on $D_{e}$, the term involving $D_{e}$ in the summation over $\mathcal{D}_{k}^{(b)}$ does not necessarily dominate the other terms.

Example 4: Consider the 16-QAM constellation. The SGHB labeling map proposed by Schreckenbach, Gortz, Hagenauer, and Bauch [5] and the HR labeling map proposed by Huang and Ritcey [6] have $D_{e}=2 d_{e 1}$ and $\xi=6.80 d_{e 1}^{2}$, and the Gray labeling map has $D_{e}=d_{e 1}$ and $\xi=1.29 d_{e 1}^{2}$. For the TV labeling map, 1000 executions of the labeling algorithm generated 806 maps with $D_{e}=\sqrt{2} d_{e 1}$ and 194 with $D_{e}=\sqrt{5} d_{e 1}$, which is the lower bound of (4). For 421 of the maps with $D_{e}=\sqrt{2} d_{e 1}, \xi=3.66 d_{e 1}^{2}$, and for the remaining 385 maps, $\xi=4.26 d_{e 1}^{2}$. For the maps with $D_{e}=\sqrt{5} d_{e 1}$, $\xi=5.90 d_{e 1}^{2}$. Thus, the TV labeling map of Fig. 2, which has $D_{e}=\sqrt{5} d_{e 1}$ and $\xi=5.90 d_{e 1}^{2}$, provides a larger $D_{e}$ but a smaller harmonic mean for 16-QAM than the SGHB and HR labeling maps. $\square$

Example 5: For the 64-QAM constellation, 16,000 TV labeling maps were generated by the constrained labeling algorithm. The TV labeling map of Fig. 3 has $D_{e}=\sqrt{13} d_{e 1}$, which is the lower bound of (4), and $\xi=23.92 d_{e 1}^{2}$, which are the largest values of $D_{e}$ and the harmonic mean among the generated TV maps. The HR labeling map for the 64QAM constellation has $D_{e}=\sqrt{17} d_{e 1}$ and $\xi=30.18 d_{e 1}^{2}$. For the 256-QAM constellation, 1300 TV labeling maps were generated by the constrained labeling algorithm. The TV labeling map of Fig. 4 has $D_{e}=\sqrt{73} d_{e 1}$, which greatly exceeds the lower bound of (4), and $\xi=102.37 d_{e 1}^{2}$, which are the largest values of $D_{e}$ and the harmonic mean among the generated TV maps. The bit labels are expressed in a decimal format, and the constellation points are not shown for simplicity.

\section{Simulation Examples}

The TV labeling maps are designed to increase the effective Euclidean distance once at least two bits are essentially known.
Therefore, they are expected to be increasingly advantageous as the number of bits per symbol increases. Simulation results confirm this characteristic. A TV labeling map maximizes the number of bits that differ between adjacent symbols. Thus, the error floor will be low, but the onset of the waterfall region will be adversely shifted relative to other labeling maps.

To illustrate the effects of the TV labeling maps, simulations were conducted to generate plots of the bit error rate (BER) of several BICM-ID systems as a function of $\mathcal{E}_{b} / N_{0}$, where $\mathcal{E}_{b}$ is the bit energy. The transmitted symbols experience independent and identically distributed Rayleigh fading. In Figures 5 and 6, coherent QAM demodulation and a rate$1 / 2$ convolutional code with constraint length $K=3$ and octal generators $(5,7)$ are used. The input to the convolutional encoder comprises 1200 information bits followed by 2 tail bits, and the resulting codeword of length 2404 code bits is passed through the interleaver.

Figure 5 shows the results for 64-QAM with 5 or 10 iterations of the demodulator and decoder. The labeling maps are the TV map of Fig. 3, the HR map, and the Gray map. All but the Gray labeling map are designed to produce a low error floor in a BICM-ID system. Although the Gray labeling map is advantageous for BICM, it is distinctly inferior to the other labeling maps for BICM-ID and bit error rates below $10^{-3}$. Despite its lower harmonic mean, the TV labeling map provides a lower error floor and a better performance than the HR labeling map when the bit error rate is below $10^{-7}$. The error-free feedback (EFF) bounds [1], [2], which assume that the demodulator is provided with ideal feedback from the decoder, were computed by the method of residues. As shown in the figure, the EFF bound provides a much more accurate error-floor prediction for the TV map than for the HR map. Thus, neither the harmonic mean nor the EFF bound accurately predicts the relative error floors of labeling maps.

The cost of the lower error floor of the TV map in Fig. 5 is a significant degradation in the waterfall region relative to the performance of the HR map. This example indicates that the labeling map that provides the lowest error floor may not be as desirable as another map in terms of overall performance, which may be more important than the error floor at high SNR. Thus, in practical applications, a simulation of the waterfall region is needed to assess the relative merits of labeling maps with low error floors.

The heuristic searches required by the HR and SGHB maps are very complex and inefficient to implement for 128QAM and larger constellations, whereas the TV map is easily generated for large constellations and ensures a low error floor. Figure 6 shows the results for 256-QAM, the TV map of Fig. 4 and the Gray map, and BICM-ID with 5 iterations of the demodulator and decoder; the results for 10 iterations are nearly the same. The onset of the waterfall region for the TV map is greatly delayed relative to the Gray map, but then the plot for the TV map shows a steep descent that crosses the plot for the Gray map and continues to fall rapidly toward a very low error floor approximated by the EFF bound. A simulation of a TV map with a lower harmonic mean exhibited no detectable difference in the waterfall region.

Figure 7 shows the bit error rates for noncoherent CPFSK with $q=16, h=0.21$, a rate- $1 / 2$ convolutional code with 


\begin{tabular}{|c|c|c|c|c|c|c|c|c|c|c|c|c|c|c|c|}
\hline 0 & 239 & 24 & 199 & 120 & 151 & 40 & 223 & 48 & 143 & 96 & 191 & 80 & 167 & 72 & 247 \\
\hline 251 & 20 & 227 & 60 & 131 & 108 & 211 & 36 & 203 & 116 & 155 & 68 & 171 & 92 & 179 & 12 \\
\hline 132 & 107 & 156 & 67 & 252 & 19 & 172 & 91 & 180 & 11 & 228 & 59 & 212 & 35 & 204 & 115 \\
\hline 122 & 149 & 98 & 189 & 2 & 237 & 82 & 165 & 74 & 245 & 26 & 197 & 42 & 221 & 50 & 141 \\
\hline 129 & 110 & 153 & 70 & 249 & 22 & 169 & 94 & 177 & 14 & 225 & 62 & 209 & 38 & 201 & 18 \\
\hline 254 & 17 & 230 & 57 & 134 & 105 & 214 & 33 & 206 & 113 & 158 & 65 & 174 & 89 & 182 & \\
\hline 5 & 234 & 29 & 194 & 125 & 146 & 45 & 218 & 53 & 138 & 101 & 186 & 85 & 162 & 77 & 42 \\
\hline 248 & 23 & 224 & 63 & 128 & 111 & 208 & 39 & 200 & 119 & 152 & 71 & 168 & 95 & 176 & \\
\hline 3 & 236 & 27 & 196 & 123 & 148 & 43 & 220 & 51 & 140 & 99 & 188 & 83 & 164 & 75 & \\
\hline 253 & 18 & 229 & 58 & 133 & 106 & 213 & 34 & 205 & 114 & 157 & 66 & 173 & 90 & 181 & \\
\hline 6 & 233 & 30 & 193 & 126 & 145 & 46 & 217 & 54 & 137 & 102 & 185 & 86 & 161 & 78 & \\
\hline 121 & 150 & 97 & 190 & 1 & 238 & 81 & 166 & 73 & 246 & 25 & 198 & 41 & 222 & 49 & \\
\hline 135 & 104 & 159 & 64 & 255 & 16 & 175 & 88 & 183 & 8 & 231 & 56 & 215 & 32 & 207 & \\
\hline 124 & 147 & 100 & 187 & 4 & 235 & 84 & 163 & 76 & 243 & 28 & 195 & 44 & 219 & 52 & \\
\hline 130 & 109 & 154 & 69 & 250 & 21 & 170 & 93 & 178 & 13 & 226 & 61 & 210 & 37 & 202 & \\
\hline 127 & 144 & 103 & 184 & 7 & 232 & 87 & 160 & 79 & 240 & 31 & 192 & 47 & 216 & 55 & \\
\hline
\end{tabular}

Fig. 4. Labeling map for 256-QAM constellation.

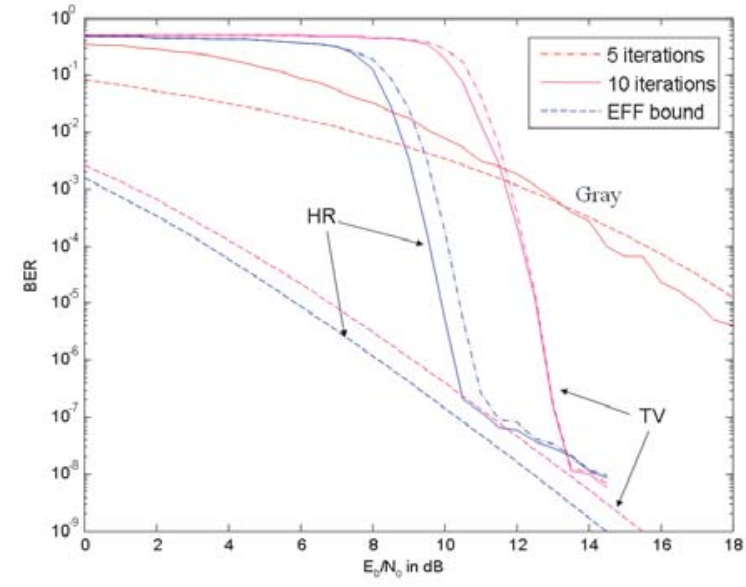

Fig. 5. Bit error rates for various 64-QAM systems with BICM-ID.

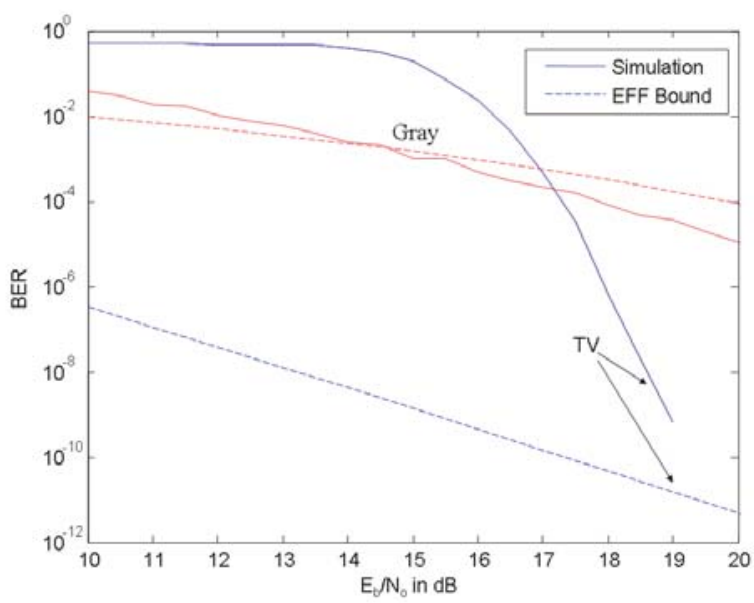

Fig. 6. Bit error rates for 256-QAM systems with BICM-ID and TV and Gray labeling maps.

constraint length $K=4$, and octal generators $(13,15)$. The input to the convolutional encoder comprises 2048 information bits followed by 3 tail bits, and the resulting codeword of length 4102 code bits is passed through the interleaver. The BICM-ID system uses 5 or 10 demodulator and decoder

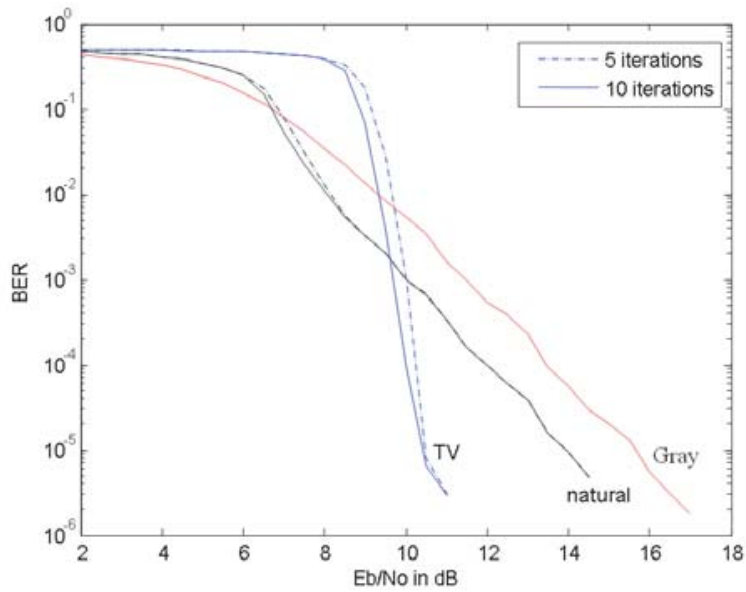

Fig. 7. Bit error rates for convolutionally coded CPFSK with BICMID, $q=16, h=0.21$, and constraint length $K=4$.

iterations and the Gray map, the natural map, or the TV map of example 1. The TV map has $\beta=3$, and the application of (3) yields $D_{e}=1.036 \sqrt{\mathcal{E}_{s}}$. The figure illustrates the dramatic lowering of the error floor when the TV map is used with BICM-ID. For bit error rates below $10^{-3}$, the TV map has an expanding advantage that is nearly $4 \mathrm{~dB}$ and $6 \mathrm{~dB}$ relative to the natural and Gray maps, respectively, when the bit error rate is $10^{-5}$.

\section{CONCLUSIONS}

The class of TV labeling maps for signal-set constellations and iterative decoding and demodulation has been derived and applied to a variety of specific communication systems that operate in the presence of ergodic Rayleigh fading. Simulation results indicate that the TV labeling maps are comparable to or better than other proposed labeling maps in providing a low error floor. The cost is an adverse shift in the onset of the waterfall region of the bit error rate. A major advantage of the TV labeling maps is that they are easily generated even when the alphabet size is large.

\section{REFERENCES}

[1] G. Caire, G. Taricco, and E. Biglieri, "Bit-interleaved coded modulation," IEEE Trans. Inform. Theory, vol. 44, pp. 927-946, May 1998. 
[2] A. Chindapol and J. A. Ritcey, "Design, analysis and performance evaluation for BICM-ID with square QAM constellations in Rayleigh fading channels," IEEE J. Select. Areas Commun., vol. 19, pp. 944-957, May 2001.

[3] X. Li, A. Chindapol, and J. A. Ritcey, "Bit-interleaved coded modulation with iterative decoding and 8PSK modulation," IEEE Trans. Commun., vol. 50, pp. 1250-1257, Aug. 2002.

[4] A. Boronka and J. Speidel, "A low complexity MIMO system based on BLAST and iterative anti-Gray-demapping," in Proc. IEEE Intern. Symp. Persona1, Indoor and Mobile Radio Commun. (PIMRC), 2003.

[5] F. Schreckenbach, N. Gortz, J. Hagenauer, and G. Bauch, "Optimization of symbol mappings for bit-interleaved coded modulation with iterative decoding," IEEE Commun. Lett., vol. 7, pp.593-595, Dec. 2003.

[6] Y. Huang and J. A. Ritcey, "Optimal constellation labeling for iteratively decoded bit-interleaved space-time coded modulation," IEEE Trans Inform. Theory, vol. 51, pp. 1865-1871, May 2005.

[7] J. Tan and G. L. Stuber, "Analysis and design of symbol mappers for iteratively decoded BICM," IEEE Trans. Wireless Commun., vol. 4, pp. 662-672, Mar. 2005.

[8] E. Rosnes and O. Ytrehus, "On the design of bit-interleaved turbo-coded modulation with low error floors," IEEE Trans. Commun., vol. 54, pp. 1563-1573, Sept. 2006.

[9] R. D. Maddock and A. H. Banihashemi, "Reliability-based coded modulation with low-density parity-check codes," IEEE Trans. Commun., vol. 54, pp. 403-406, Mar. 2006.

[10] M. C. Valenti and S. Cheng, "Iterative demodulation and decoding of turbo coded M-ary noncoherent orthogonal modulation," IEEE J. Select. Areas Commun., vol. 23, pp. 1738-1747, Sept. 2005.

[11] S. Benedetto, D. Divsalar, G. Montorsi, and F. Pollara, "A soft-input soft-output APP module for iterative decoding of concatenated codes," IEEE Commun. Lett., vol. 1, pp. 22-24, Jan. 1997.

[12] S. Pfletschinger and F. Sanzi, "Error floor removal for bit-interleaved coded modulation with iterative detection," IEEE Trans. Wireless Commun., vol. 5, pp. 3174-3181, Sept. 2006.

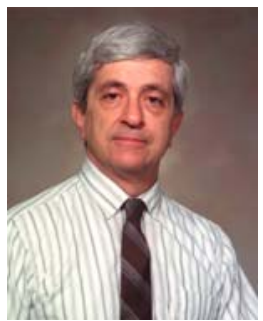

Don Torrieri is a research engineer and Fellow of the US Army Research Laboratory. His primary research interests are communication systems, adaptive arrays, and signal processing. He received the $\mathrm{Ph}$. D. degree from the University of Maryland. He is the author of many articles and several books including Principles of Spread-Spectrum Communication Systems (Springer, 2005). He teaches graduate courses at Johns Hopkins University and has taught many short courses. In 2004, he received the Military Communications Conference achievement award for sustained contributions to the field.

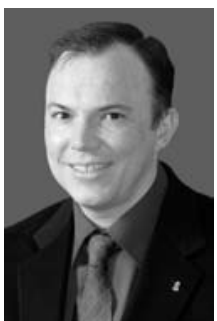

Matthew C. Valenti holds $\mathrm{BS}$ and $\mathrm{Ph} . \mathrm{D}$. degrees in Electrical Engineering from Virginia Tech. From 1992 to 1995 he was an electronics engineer at the US Naval Research Laboratory, at which time he earned a MS in electrical engineering from the Johns Hopkins University. Since 1999, he has been with West Virginia University, where he is currently an Associate Professor in the Lane Department of Computer Science and Electrical Engineering. He serves as an associate editor for IEEE TRANSACTIONS ON WIRELESS COMMUNICATIONS, and has served as a track co-chair for the Fall 2007 Vehicular Technology Conference (Baltimore, Maryland) and the 2009 International Conference on Communications (Dresden, Germany). His research interests are in the areas of communication theory, error correction coding, applied information theory, and wireless networks. 